\section{What about the role of sulfasalazine?}

Although we agree with Klassen and colleagues $^{1}$ that their patient's acute interstitial nephritis was most likely caused by pantoprazole, we note that the patient was also on sulfasalazine.

Several case reports in inflammatory bowel disease populations have identified an association with acute interstitial nephritis and sulfasalazine use, as well as with other 5-aminosalicylate (5-ASA) moieties. ${ }^{2.3}$ A mean overall nephrotoxicity rate of $0.3 \%$ has been identified in 5-ASA trials in inflammatory bowel disease, comprising 2671 patients over a total of 3070 person-years of follow-up. ${ }^{2}$ Nephrotoxicity in this population usually presented as interstitial nephritis. ${ }^{4}$ The American College of Gastroenterology's Ulcerative Colitis Practice Guidelines in Adults recommend serum creatinine measurements prior to and periodically during treatment with a 5-ASA moiety. ${ }^{4}$

Although more case reports identify acute interstitial nephritis with mesalazine than with sulfasalazine, data from observational studies suggest that the nephrotoxicity potential of mesalazine and sulfasalazine is similar. ${ }^{2}$ 5-ASAinduced acute interstitial nephritis is most frequently reported in the first 12 months of use, but delayed presentations several years after drug initiation have been reported. ${ }^{2}$ 5-ASA-induced acute interstitial nephritis apparently is the result of an idiosyncratic rather than a dose-responserelated reaction. ${ }^{2}$ 5-ASA withdrawal, especially if done early, results in renal recovery in the majority of patients. ${ }^{2}$

Although the patient in the case by Klassen and colleagues ${ }^{1}$ had presumably been receiving sulfasalazine for longer than pantoprazole, and pantoprazole was discontinued after sulfasalazine, both drugs have been implicated in acute interstitial nephritis. Because this information is a minor confounder, it may downgrade the calculated Naranjo score. Although pantoprazole remains the most likely culprit in this case ${ }^{1}$, acute interstitial nephritis may have been caused by a delayed reaction to sulfasalazine. Acute interstitial nephritis is a serious adverse event where early recognition and discontinuation of culprit drugs, like pantoprazole and sulfasalazine, can improve outcomes.

\section{Callum A Dargavel MD, Kurram Khan MSc MD}

Division of Gastroenterology, Department of Medicine, McMaster University, Hamilton, Ont.

\section{References}

1. Klassen S, Krepinsky LC, Prebtani AP. Pantoprazoleinduced acute interstitial nephritis. CMAJ 2013; 185:56-9.

2. Gisbert JP, Gonzalez-Lima Y, Mate J. 5-aminosalicylates and renal function in inflammatory bowel disease: a systematic review. Inflamm Bowel Dis 2007;13:629-38

3. Alivanis P, Aperis G, Lambrianou F, et al. Reversal of refractory sulfasalazine-related renal failure after treatment with corticosteroids. Clin Ther 2010;32: 1906-10.

4. Kornbluth A, Sachar DB. Practice Parameters Committee of the American College of Gastoenterology. Ulcerative colitis practice guidelines in adults: American College of Gastroenterology, Practice Parameters Committee. Am J Gastroenterol 2010;105:501-23.

CMAJ 2013. DOI:10.1503/cmaj.113-2119

\section{Dementia and history of trauma}

Regarding the CMAJ Salon article, "What my 5-year-old son taught me about medicine,"' I commend Dodek's sensitivity to his son's visit with Anni at the nursing home. I wonder however, if there weren't more to their gentle interaction than met the eye. While studying for my master's degree in counselling, I completed two practicums concurrently, one in a trauma therapy certification program and the other as a counselling assistant at a residence for patients with Alzheimer disease or other types of dementia.

One resident with advanced dementia was generally unapproachable, withdrawn and uncommunicative. During the Christmas season, a group of children arrived to sing carols at the facility. She became very agitated and tried, quite violently, to push the children out of the room. The assumption by staff was that, in her dementia and old age, she did not like children. Upon reading the social history of the patient, I discovered that as a young woman she had lived in a war-torn country. One day while walking in her village, she saw a class of children out with their teacher on a field trip. They began to cross the bridge that she herself had just traversed. Suddenly, there was a huge explosion behind her. When she looked back, the bridge had been bombed; the children and their teacher all died in the event.

This story led me to more and more research of the trauma history of many of the residents and, almost without exception, I came to the conclusion that much of their repetitive, often violent behaviour was the result of previously experienced traumatic events. In their dementia they continued to act out, appearing to attempt to resolve and heal the emotional scars of these earlier experiences. This woman, I believe, was still desperately trying to keep the children safe. To my knowledge, little research has yet to be done on the possibility that dementia, in particular Alzheimer disease, can be the result of long-term dysregulation of nervous system biochemistry in the body and the resulting neurological deficits. Dodek's story, I am certain, reflects the history of his patient Anni. Did Anni see something in Max that no one else could see - perhaps her past as a tender and loving mother who had lost her child many years before? Further research leading to a deeper understanding of the connection between unresolved life trauma and some types of dementia would perhaps lead to more compassionate therapeutic care for patients with dementia.

\section{Susan D. Russell BA MEd}

Registered clinical counsellor, British Columbia Association of Clinical

Counsellors, Victoria, BC

\section{Reference}

1. Dodek DY. What my 5-year-old son taught me about medicine. CMAJ 2013;185:536.

CMAJ 2013. DOI:10.1503/cmaj.113-2121

\section{Urethral discharge}

I would like to congratulate Rebick and colleagues $^{1}$ on their article on the treatment of urethral infections. In their elegant work, the authors describe the tests available for specific diagnosis of various causes of urethritis in men and conclude 